\title{
Base manifolds for fibrations of projective irreducible symplectic manifolds
}

\author{
Jun-Muk Hwang ${ }^{1}$
}

\begin{abstract}
Given a projective irreducible symplectic manifold $M$ of dimension $2 n$, a projective manifold $X$ and a surjective holomorphic map $f: M \rightarrow X$ with connected fibers of positive dimension, we prove that $X$ is biholomorphic to the projective space of dimension $n$. The proof is obtained by exploiting two geometric structures at general points of $X$ : the affine structure arising from the action variables of the Lagrangian fibration $f$ and the structure defined by the variety of minimal rational tangents on the Fano manifold $X$.
\end{abstract}

Key words: holomorphic Lagrangian fibration, holomorphic symplectic manifolds, variety of minimal rational tangents

2000MSC:14J40, 14J45

\section{Introduction}

We work in the category of complex analytic sets. A connected complex manifold $M$ of dimension $2 n$ equipped with a holomorphic symplectic from $\omega \in H^{0}\left(M, \Omega_{M}^{2}\right)$ is called a holomorphic symplectic manifold. A subvariety $V$ of $M$ is said to be Lagrangian if $V$ has dimension $n$ and the restriction of $\omega$ on the smooth part of $V$ is identically zero. A simply connected projective algebraic manifold $M$ is called a projective irreducible symplectic manifold if $M$ has a symplectic form $\omega$ such that $H^{0}\left(M, \Omega_{M}^{2}\right)=\mathbf{C} \omega$. It is remarkable that fibrations of projective irreducible symplectic manifolds are of very special form, as described in the following theorem due to D. Matsushita.

Theorem 1.1 Let $M$ be a projective irreducible symplectic manifold of dimension $2 n$. For a projective manifold $X$ and a surjective holomorphic map $f: M \rightarrow X$ with connected fibers of positive dimension, the following holds.

(1) $X$ is a Fano manifold of dimension $n$ with Picard number 1.

(2) A general fiber of $f$ is biholomorphic to an abelian variety.

(3) The underlying subvariety of every fiber of $f$ is Lagrangian.

(4) All even Betti numbers of $X$ are equal to 1 and all odd Betti numbers of $X$ are equal to 0 .

(1), (2) and (3) in Theorem 1.1 were proved in [Ma1] and [Ma2]. These results led to the question whether the base manifold $X$ is the complex projective space (cf. [Hu, 21.4]). The

\footnotetext{
${ }^{1}$ This work was supported by the Korea Research Foundation Grant funded by the Korean Government(MOEHRD)(KRF-2006-341-C00004).
} 
result of [Ma3] verifies Theorem 1.1 (4), i.e., that the Betti numbers of $X$ are indeed equal to those of $\mathbf{P}_{n}$. Some special cases of this question were studied in [CMS, Section 7] and [Ng].

Our goal is to give an affirmative answer to the question as follows.

Theorem 1.2 In the setting of Theorem 1.1, $X$ is biholomorphic to $\mathbf{P}_{n}$.

There are two geometric ingredients in the proof of Theorem 1.2: the theory of varieties of minimal rational tangents and the theory of Lagrangian fibrations. On the one hand, the theory of varieties of minimal rational tangents describes a certain geometric structure arising from minimal rational curves at general points of a Fano manifold $X$ with $b_{2}(X)=1$ (cf. [HwMo1], [HwMo2]). This geometric structure has differential geometric properties reflecting special features of the deformation theory of minimal rational curves. On the other hand, the theory of Lagrangian fibrations, or equivalently, the theory of completely integrable Hamiltonian systems, provides an affine structure at general points of the base manifold $X$ via the classical action variables (cf. [GuSt, Section 44]). Our strategy to prove Theorem 1.2 is to exploit the interplay of these two geometric structures on the base manifold $X$. Under the assumption that $X$ is different from $\mathbf{P}_{n}$, the condition $b_{2}(X)=1$ forces the geometric structure arising from the variety of minimal rational tangents to be 'non-flat', while the affine structure arising from the action variables is naturally 'flat'. These two structures interact via the monodromy of the Lagrangian fibration, leading to a contradiction. To be precise, two separate arguments are needed depending on whether the dimension $p$ of the variety of minimal rational tangents is positive or zero. The easier case of $p>0$ is handled by a topological argument using $b_{4}(X)=1$. The more difficult case of $p=0$ needs a deeper argument, depending on the local differential geometry of the variety of minimal rational tangents.

It is expected that an analog of Theorem 1.2 holds under the weaker assumption that $M$ is a Kähler irreducible symplectic manifold and $X$ is a compact Kähler manifold (cf. [Hu,24.8]). As far as we see, the only point where our proof of Theorem 1.2 uses the projectivity assumption in an essential way is the result $b_{4}(X)=1$ from [Ma3], which is used to handle the case of $p>0$. Thus if [Ma3] is generalizable to Kähler setting, so is our result. On the other hand, after seeing the first draft of this paper, D. Matsushita informed us that he has a new approach to the case of $p>0$, which may lead to the generalization to Kähler setting, even allowing some singularity on $M$.

It is also expected that an analog of Theorem 1.2 holds under the weaker assumption that $X$ is a normal projective variety. In fact, under this weaker assumption, [Ma1] showed that the singularity of $X$ is mild and $X$ must be a Fano variety of Picard number 1 . However, our approach to Theorem 1.2 uses the smoothness of $X$ in a crucial way and seems difficult to generalize to the singular setting.

In Section 2, we present results about varieties of minimal rational tangents of Fano manifolds that are independent of Lagrangian fibrations. In Section 3, we present results about Lagrangian fibrations that are independent of Fano manifolds. These two theories are played against each other to prove Theorem 1.2, first in the case of $p>0$ in Section 4 and then in the case of $p=0$ in Section 5. 


\section{Results on varieties of minimal rational tangents}

In this section, we present several results about varieties of minimal rational tangents, especially when they are linear. Most of these are already known, for which we only give brief explanation with precise references.

Throughout this section, we will denote by $X$ an $n$-dimensional Fano manifold with $b_{2}(X)=1$. An irreducible component $\mathcal{K}$ of the space of rational curves on $X$ is called a minimal component if for a general point $x \in X$, the subscheme $\mathcal{K}_{x}$ of $\mathcal{K}$ consisting of members passing through $x$ is non-empty and complete. In this case, the subvariety $\mathcal{C}_{x}$ of the projectivized tangent space $\mathbf{P} T_{x}(X)$ consisting of the tangent directions at $x$ of members of $\mathcal{K}_{x}$ is called the variety of minimal rational tangents at $x$ (see [HwMo2] for more details). For a general member $C$ of $\mathcal{K}$, the normalization $\nu: \hat{C} \rightarrow C \subset X$ is an immersion of $\mathbf{P}_{1}$.

The following result is proved in [HwRa, Corollary 2.2].

Proposition 2.1 Given a minimal component $\mathcal{K}$ on $X$ and a general point $x \in X$, let $\mathcal{C}_{1}$ be a component of $\mathcal{C}_{x}$. Denote by $\mathcal{K}_{1}$ the corresponding component of $\mathcal{K}_{x}$. For a general member $C$ of $\mathcal{K}_{1}$, let $S_{C} \subset \mathbf{P} T_{x}^{*}(X)$ be the linear subspace of the projectivized cotangent space that is the image of the evaluation of $H^{0}\left(\hat{C}, \nu^{*} T^{*}(X)\right)$ at $x$. Then the closure of the union of $S_{C}$ as $C$ varies over general points of $\mathcal{K}_{1}$ is the dual variety of $\mathcal{C}_{1} \subset \mathbf{P} T_{x}(X)$.

Proposition 2.1 is useful when combined with the following.

Proposition 2.2 Given a minimal component $\mathcal{K}$ on $X$ and a general point $x \in X$, suppose the dual variety $\mathcal{C}_{1}^{*} \subset \mathbf{P} T_{x}^{*}(X)$ of a component $\mathcal{C}_{1}$ of $\mathcal{C}_{x}$ is linearly degenerate, i.e., contained in a hyperplane in $\mathbf{P} T_{x}^{*}(X)$. Then the component $\mathcal{C}_{1}$ is a linear subspace of $\mathbf{P} T_{x}(X)$.

Proof. If $\mathcal{C}_{1}^{*}$ is linearly degenerate in $\mathbf{P} T_{x}^{*}(X)$, then $\mathcal{C}_{1}$ is a cone. Thus Proposition 2.2 is equivalent to [HwMo2, Proposition 13], which says that $\mathcal{C}_{1}$ cannot be a cone unless it is a linear subspace.

The next proposition is [HwMo1, Lemma 4.2].

Proposition 2.3 Let $X^{\prime}$ be a projective algebraic manifold and $X$ be a Fano manifold with $b_{2}(X)=1$. Fix a minimal component $\mathcal{K}$ on $X$. Given a generically finite morphism $\mu: X^{\prime} \rightarrow X$ which is not birational, and given a general member $C \subset X$ of $\mathcal{K}$, there exists a component $C^{\prime}$ of $\mu^{-1}(C)$ such that the restriction $\left.\mu\right|_{C^{\prime}}: C^{\prime} \rightarrow C$ is finite of degree $>1$.

For the rest of this section, we will make the following assumption.

(Assumption) $X$ is an $n$-dimensional Fano manifold with $b_{2}=1$, different from $\mathbf{P}_{n}$, and for some choice of $\mathcal{K}$, the variety of minimal rational tangents at a general point is the union of linear subspaces of dimension $p \geq 0$.

The condition $X \neq \mathbf{P}_{n}$ implies that $p<n-1$ by [CMS, Theorem 0.2] and the condition $b_{2}(X)=1$ implies that $\mathcal{C}_{x}$ has at least two irreducible components. In an analytic local neighborhood of $x$, each component of $\mathcal{C}_{x}$ defines a distribution. It is easy to see that this distribution is integrable and the leaf through $x$ is an immersed $\mathbf{P}_{p+1}$. More precisely, we have the following result from $[\mathrm{Hw}$, Proposition 1]. 
Proposition 2.4 When $X$ satisfies the above assumption, there exists a projective algebraic manifold $X^{\prime}$ with a generically finite holomorphic map $\mu: X^{\prime} \rightarrow X$ of degree $>1$ and a proper holomorphic map $\rho: X^{\prime} \rightarrow Z$ onto a positive-dimensional projective manifold $Z$ such that $\rho$ is a $\mathbf{P}_{p+1}$-bundle over a Zariski open subset $Z_{o} \subset Z, \mu$ is unramified on $\rho^{-1}\left(Z_{o}\right)$, and each member of $\mathcal{K}_{x}$ for a general $x \in X$ is the image of a line in some $\mathbf{P}_{p+1}$-fibers of $\rho$. Here, $p+\operatorname{dim} Z=n-1$ and $\operatorname{dim} Z=\operatorname{dim} H^{0}\left(\hat{C}, \nu^{*} T^{*}(X)\right)$ for the normalization $\nu: \hat{C} \rightarrow C \subset X$ of a general member $C$ of $\mathcal{K}$. Moreover, if we set $P_{\zeta}:=\mu\left(\rho^{-1}(\zeta)\right)$ for each $\zeta \in Z_{o}$, then the following holds.

(a) $P_{\zeta}$ is an immersed submanifold with trivial normal bundle in $X$.

(b) $\left.\mu\right|_{\rho^{-1}(\zeta)}$ is the normalization of $P_{\zeta}$.

(c) For two distinct points $\zeta_{1} \neq \zeta_{2} \in Z_{o}$, the two subvarieties $P_{\zeta_{1}}$ and $P_{\zeta_{2}}$ are distinct.

Here, when $X$ is a complex manifold and $V \subset X$ is a subvariety, we say that $V$ is an immersed submanifold if the normalization $\hat{V}$ is smooth and the normalization map $\nu: \hat{V} \rightarrow V \subset X$ is an immersion. The normal bundle of $V$ means the vector bundle on $\hat{V}$ defined as the quotient of $\nu^{*} T(X)$ by the image of $T(\hat{V})$. If the normal bundle of $V$ is a trivial bundle, we say that $V$ is an immersed submanifold with trivial normal bundle.

The first sentence of the following proposition is exactly [Hw, Proposition 2.2], from which the second sentence follows because $P_{\zeta}, \zeta \in Z_{o}$, is immersed with trivial normal bundle.

Proposition 2.5 In the setting of Proposition 2.4, for a general point $x \in X$, the variety of minimal rational tangents $\mathcal{C}_{x}$ is a disjoint union of linear subspaces in $\mathbf{P} T_{x}(X)$, i.e., for each $\zeta_{1} \neq \zeta_{2} \in Z_{o}$ with $x \in P_{\zeta_{1}} \cap P_{\zeta_{2}}$, the intersection of the tangent spaces $T_{x}\left(P_{\zeta_{1}}\right)$ and $T_{x}\left(P_{\zeta_{2}}\right)$ in $T_{x}(X)$ is zero. Consequently, the elements of $T_{x}^{*}(X)$ obtained by evaluating $H^{0}\left(\rho^{-1}\left(\zeta_{1}\right), \mu^{*} T^{*}(X)\right)$ and $H^{0}\left(\rho^{-1}\left(\zeta_{2}\right), \mu^{*} T^{*}(X)\right)$ at $x$ span $T_{x}^{*}(X)$.

In the situation of Proposition 2.4, it is convenient to introduce the following notion, which already played an essential role in $[\mathrm{Hw}]$. We say that $\mathcal{K}$ is multivalent on an irreducible hypersurface $H \subset X$, if the following holds: when $H^{\prime} \subset X^{\prime}$ denotes the union of the components of $\mu^{-1}(H)$ that are dominant over $H$ by $\mu$ and dominant over $Z$ by $\rho$, the dominant map $H^{\prime} \rightarrow H$ has degree $>1$. The next proposition is a variation of [Hw, Proposition 3.2].

Proposition 2.6 In the setting of Proposition 2.4, suppose that $\mathcal{K}$ is multivalent on an irreducible hypersurface $H \subset X$. Then a general point $y \in H$ has two open neighborhoods $W \subset W_{0}$ satisfying the following.

(1) The fundamental group $\pi_{1}\left(W_{0} \backslash H\right)$ is cyclic.

(2) For each general point $x \in W \backslash H$, there are two distinct points $\zeta_{1}, \zeta_{2} \in Z_{o}$ with $x \in P_{\zeta_{1}} \cap P_{\zeta_{2}}$ and $T_{x}\left(P_{\zeta_{1}}\right) \cap T_{x}\left(P_{\zeta_{2}}\right)=0$ such that there exists a loop $\gamma_{1}$ (resp. $\gamma_{2}$ ) based at $x$ lying on the smooth locus of $P_{\zeta_{1}} \backslash H$ (resp. $P_{\zeta_{2}} \backslash H$ ), the homotopy class of which generates the cyclic group $\pi_{1}\left(W_{0} \backslash H, x\right)$.

Proof. The multivalence assumption on $H$ implies that there are two distinct $P_{\zeta_{3}}$ and $P_{\zeta_{4}}$ passing through $y$. The desired $P_{\zeta_{1}}$ and $P_{\zeta_{2}}$ can be obtained as small deformations of $P_{\zeta_{3}}$ and $P_{\zeta_{4}}$. Let us make this more precise.

Since $\mathcal{K}$ is multivalent on $H$, there exist two distinct points $y_{1}, y_{2}$ in $\mu^{-1}(y) \cap H^{\prime}$ such that the map $\mu$ is unramified at $y_{1}$ and $y_{2}$. We can choose open neighborhoods $W_{1} \subset X^{\prime}$ of $y_{1}, W_{2} \subset X^{\prime}$ of $y_{2}$ and $W_{0} \subset X$ of $y$ with the following properties: 
(a) $W_{0}$ is biholomorphic to the polydisc $\Delta^{n}$ and $W_{0} \cap H$ corresponds to the coordinate hyperplane $\Delta^{n-1} \subset \Delta^{n}$.

(b) $\mu\left(W_{1}\right)=\mu\left(W_{2}\right)=W_{0}$

(c) $\left.\mu\right|_{W_{1}}$ and $\left.\mu\right|_{W_{2}}$ are biholomorphic over $W_{0}$.

(d) $W_{1} \cap H^{\prime}$ and $W_{2} \cap H^{\prime}$ are transversal to fibers of $\rho$ at the intersection points, i.e., their scheme-theoretic intersections with the fibers of $\rho$ are smooth.

We can assume that $\rho$ is a $\mathbf{P}_{p+1}$-bundle near $\rho\left(y_{1}\right)$ (resp. $\rho\left(y_{2}\right)$ ), so there exists an open neighborhood $W_{1}^{\prime} \subset W_{1}$ of $y_{1}$ (resp. $W_{2}^{\prime} \subset W_{2}$ of $y_{2}$ ) such that for any $w \in W_{1}^{\prime}$ (resp. $w \in W_{2}^{\prime}$ ),

$\rho^{-1}(\rho(w)) \cap W_{1}^{\prime}$ (resp. $\rho^{-1}(\rho(w)) \cap W_{2}^{\prime}$ ) is irreducible. Now choose $W$ to be a neighborhood of $y$ in $\mu\left(W_{1}^{\prime}\right) \cap \mu\left(W_{2}^{\prime}\right)$. Then (1) is automatic and (2) can be seen from the following lemma.

Lemma 2.7 Let $B=\Delta^{n}$ and $H \subset B$ be the coordinate hyperplane $\Delta^{n-1} \subset \Delta^{n}$. Let $y \in H$ be a point and $V \subset B$ be an irreducible closed immersed submanifold in $B$ such that $y \in V$ and an irreducible component of the germ of $V$ at $y$ is non-singular and intersects $H$ transversally at $y$. Then for any non-singular point $x$ of $V$, there exists a loop based at $x$ lying on the smooth locus of $V \backslash H$ which generates the cyclic fundamental group $\pi_{1}(B \backslash H, x)$.

Proof. Since $V$ is irreducible, it suffices to show this for some non-singular point $x \in V \backslash H$. But this is obvious if $x$ lies on the irreducible component of the germ of $V$ intersecting $H$ transversally at $x$.

The next proposition is precisely [Hw, Proposition 3.1].

Proposition 2.8 Let $X$ be a Fano manifold with $b_{2}(X)=b_{4}(X)=1$ satisfying the assumption of Proposition 2.4 with $p>0$. Then the minimal component $\mathcal{K}$ is multivalent on every irreducible hypersurface $H \subset X$.

The condition $p>0$ in Proposition 2.8 is crucial. In fact, when $X$ is a Fano 3-fold defined by a linear section of the 6-dimensional Grassmannian of rank 3, there is a surface $H \subset X$ such that the minimal component $\mathcal{K}$ is not multivalent on $H$.

\section{Results on Lagrangian fibrations}

In this section, we present some results about Lagrangian fibrations.

In this paper, the phrase 'Lagrangian fibration' will have the following restrictive meaning. Let $(M, \omega)$ be a holomorphic symplectic manifold and $B$ be a complex manifold of dimension $n$. A proper surjective holomorphic map $f: M \rightarrow B$ is a Lagrangian fibration if it satisfies the statements (2) and (3) of Theorem 1.1, i.e., if a general fiber of $f$ is biholomorphic to an abelian variety and the underlying subvariety of every fiber of $f$ is Lagrangian.

Given a Lagrangian fibration $f: M \rightarrow B$, the locus $D \subset B$ of the critical values of $f$ is a hypersurface (e.g. [HO, Proposition 3.1]), if non-empty. We will call $D$ the critical set of $f$. The following is well-known, see, for example, [HO, Proposition 3.2] for a proof.

Proposition 3.1 Let $f: M \rightarrow B$ be a Lagrangian fibration. Given $b \in B$, if the underlying reduced variety of $f^{-1}(b)$ is an abelian variety, then $f^{-1}(b)$ is smooth, i.e., $b$ is not in the critical set of $f$. 
A proper holomorphic map $f: M \rightarrow B$ between two connected complex manifolds is called a smooth abelian fibration if every fiber is biholomorphic to an abelian variety. Recall that for a smooth abelian fibration, a choice of a base point $b \in B$ gives rise to the monodromy representation

$$
\pi_{1}(B, b) \longrightarrow \mathrm{GL}\left(H_{1}\left(f^{-1}(b), \mathbf{Z}\right)\right) .
$$

The following result is due to Matsushita [Ma4]. Since its proof in [Ma4] is buried in a longer argument to prove a much more substantial result, we will give a sketch of the proof for readers' convenience.

Proposition 3.2 Let $f: M \rightarrow B$ be a Lagrangian fibration with a non-empty critical set $D \subset B$. Assume that $B$ is biholomorphic to the polydisc $\Delta^{n}$ and the critical set $D \subset B$ as a subvariety is biholomorphic to a coordinate hyperplane $\Delta^{n-1} \subset \Delta^{n}$. Assume furthermore that there exists a line bundle on $M$ which is ample on each fiber. Then the monodromy of the smooth abelian fibration $M \backslash f^{-1}(D) \rightarrow B \backslash D$ is non-trivial, i.e., for any point $b \in B \backslash D$, the image of the representation

$$
\pi_{1}(B \backslash D, b) \longrightarrow \mathrm{GL}\left(H_{1}\left(f^{-1}(b), \mathbf{Z}\right)\right)
$$

is not the identity.

Proof. Assume that the monodromy is trivial. We can choose a cyclic cover of $B$ along $D$, $\xi: B^{\prime} \rightarrow B$ such that the induced fibration $\xi^{*} f: \xi^{*} M \rightarrow B^{\prime}$ has a section. If $f$ already has a section, we choose $\xi$ to be the identity map of $B$. Let $D^{\prime}=\xi^{-1}(D)_{\text {red }}$ be the ramification set of $\xi$. Using the triviality of the monodromy of $\xi^{*} f$ and the ample line bundle, we can define the period map from $B^{\prime} \backslash D^{\prime}$ to the Siegel upper half space, which extends to a holomorphic map from $B^{\prime}$ to the Siegel upper half space. Using the extended period map, we can construct a smooth abelian fibration $\tilde{f}: \tilde{M} \rightarrow B^{\prime}$ with a biholomorphic map

$$
\Phi: \xi^{*} M \backslash\left[\left(\mu^{*} f\right)^{-1}\left(D^{\prime}\right)\right] \longrightarrow \tilde{M} \backslash \tilde{f}^{-1}\left(D^{\prime}\right) .
$$

The existence of the section for $\xi^{*} f$ ensures that $\Phi$ defines a bimeromorphic map between $\xi^{*} M$ and $\tilde{M}$, by the argument of Nakayama [Nk, Proposition 1.6]. The action of the Galois group $\Gamma$ of the cyclic cover $\xi$ on $\xi^{*} M$ induces an action of $\Gamma$ on $\tilde{M}$. If $\Gamma$ is not trivial, i.e., if $f$ doesn't have a section, then $\Gamma$ acts non-trivially on the fibers of $\tilde{f}$ over $D^{\prime}$. On the other hand, $\Gamma$ acts trivially on the singular homology group of the fibers of $\tilde{f}$ over $D^{\prime}$ by the triviality of the monodromy. This means that $\Gamma$ acts as a translation on the fibers of $\tilde{f}$ over $D^{\prime}$. Thus the quotient of $\tilde{M}$ by $\Gamma$ is an abelian fibration $\tilde{M} / \Gamma \rightarrow B$, the underlying reduced variety of each fiber of which is an abelian variety. In particular, $\tilde{M} / \Gamma$ contains no rational curve. The bimeromorphic map $\Phi$ descends to a bimeromorphic map between $M$ and $\tilde{M} / \Gamma$. Since $\hat{M} / \Gamma$ contains no rational curve, $M$ and $\hat{M}$ are biholomorphic. By Proposition 3.1, the critical set of $f$ is empty, a contradiction.

Given a holomorphic symplectic manifold $(M, \omega)$, the contraction with $\omega$ induces a natural isomorphism

$$
\iota_{\omega}: T^{*}(M) \longrightarrow T(M) .
$$

For a Lagrangian fibration $f: M \rightarrow B$, any $b \in B$ and any $z \in f^{-1}(b)$, we have a homomorphism

$$
\iota_{\omega} \circ f^{*}: T_{b}^{*}(B) \longrightarrow T_{z}(M) .
$$


If $z$ is a non-singular point of $f^{-1}(b)$, this induces an isomorphism

$$
\iota_{b, z}: T_{b}^{*}(B) \longrightarrow T_{z}\left(f^{-1}(b)\right) .
$$

If $b$ is not in the critical set, denote by $\operatorname{Aut}_{o}\left(f^{-1}(b)\right)$ the identity component of the automorphism group of the abelian variety $f^{-1}(b)$. Then for any $z \in f^{-1}(b), \iota_{b, z}$ induces a natural unramified surjective group homomorphism

$$
h_{b}: T_{b}^{*}(B) \longrightarrow \operatorname{Aut}_{o}\left(f^{-1}(b)\right) .
$$

An analog of this exists for arbitrary $b \in B$ as follows.

Proposition 3.3 Let $f: M \rightarrow B$ be a Lagrangian fibration. For each point $b \in B$, there exists a canonical homomorphism of complex Lie groups

$$
h_{b}: T_{b}^{*}(B) \longrightarrow \operatorname{Aut}_{o}\left(f^{-1}(b)_{\mathrm{red}}\right),
$$

where $T_{b}^{*}(B)$ is the vector group and the target is the identity component of the automorphism group of the underlying reduced variety of the fiber at $b$.

Proof. For each $v \in T_{b}^{*}(B)$, let $\tilde{v}$ be an exact 1 -form in a local analytic neighborhood $U$ of $b$ which coincides with $v$ at $b$. The vector field $\iota_{\omega}\left(f^{*} \tilde{v}\right)$ on $f^{-1}(U)$ is a Hamiltonian vector field and is tangent to the fibers of $f$. Thus it induces a derivation $\vec{v}$ on $f^{-1}(b)_{\text {red. }}$. At a point $z$ of $f^{-1}(b)_{\text {red }}$, $\vec{v}$ is just $\iota_{\omega} \circ f^{*}(v)$. Thus this derivation is independent of the choice of $\tilde{v}$ and depends only on $v$. By integrating the derivation (e.g. [Ka, p.83, Korollar]), we get a natural group homomorphism $\mathbf{C} v \rightarrow \operatorname{Aut}_{o}\left(f^{-1}(b)_{\text {red }}\right)$. This defines $h_{b}: T_{b}^{*}(B) \rightarrow \operatorname{Aut}_{o}\left(f^{-1}(b)_{\text {red }}\right)$ in a canonical way.

Proposition 3.4 Let $f: M \rightarrow B$ be a Lagrangian fibration and let $V$ be an irreducible complete variety in $B$. Then the following holds.

(i) There exists a canonical homomorphism of complex Lie groups

$$
h_{V}: H^{0}\left(V, T^{*}(B)\right) \longrightarrow \operatorname{Aut}_{o}^{f}\left(f^{-1}(V)_{\text {red }}\right)
$$

where the target denotes the identity component of the complex Lie group of automorphisms preserving the $f$-fibers of the underlying reduced variety of $f^{-1}(V)$.

(ii) If $V$ is not contained in the critical set of $f$, the group $\operatorname{Aut}_{o}^{f}\left(f^{-1}(V)_{\text {red }}\right)$ is an abelian variety, which we denote by $A_{V}$.

(iii) If $V$ is not contained in the critical set of $f$, but has a non-empty intersection with the critical set, then the abelian variety $A_{V}$ has dimension $\leq n-1$.

(iv) Suppose that $b \in V$ is not in the critical set and $z \in f^{-1}(b)$. Let $T_{e}\left(A_{V}\right)$ be the tangent space of the abelian variety $A_{V}$ in (ii) at the identity. The homomorphism $h_{V}$ in (i) induces

$$
d h_{V}: H^{0}\left(V, T^{*}(B)\right) \longrightarrow T_{e}\left(A_{V}\right)
$$

and there is a commutative diagram

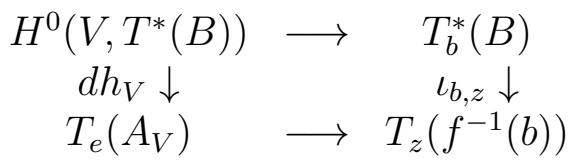


where the upper horizontal arrow is the evaluation at $b$ and the lower horizontal arrow is the tangent map for the $A_{V}$-orbit of $z$.

Proof. For each $\xi \in H^{0}\left(V, T^{*}(B)\right)$, the automorphism $h_{V}(\xi)$ is defined such that it acts on $y \in f^{-1}(V)_{\text {red }}$ by

$$
h_{V}(\xi) \cdot y=h_{b}\left(\xi_{b}\right) \cdot y
$$

where $b=f(y) \in V, h_{b}$ is the homomorphism defined in Proposition 3.3 and $\xi_{b}$ is the value of $\xi$ at $b$. This gives (i). To see (ii), suppose that the algebraic group $\operatorname{Aut}_{o}^{f}\left(f^{-1}(V)_{\text {red }}\right)$ is not an abelian variety. Then it contains a linear algebraic subgroup by Chevalley decomposition. This linear algebraic group must act non-trivially on $f^{-1}(b)$ for a general point $b \in V$. But linear algebraic groups cannot act on abelian varieties nontrivially, a contradiction. Regarding (iii), if the dimension of $\operatorname{Aut}_{o}^{f}\left(f^{-1}(V)_{\text {red }}\right)$ is $\geq n$, the fiber of $f$ over each point of $V$ contains an orbit, which must be an abelian variety of dimension $\geq n$. Thus each fiber is smooth by Proposition 3.1, a contradiction to the assumption in (iii). Finally, (iv) is immediate from the definition.

Recall that the cotangent bundle of a complex manifold has a canonical holomorphic symplectic form on it. The following is a geometric version of the classical action-angle variables for an integrable Hamiltonian system. The proof in [GS, Theorem 44.2] works verbatim in the holomorphic setting.

Proposition 3.5 Let $f: M \rightarrow B$ be a smooth Lagrangian fibration with a Lagrangian section $\Sigma \subset M$. The orbit of $\Sigma$ under the action of $T_{b}^{*}(B), b \in B$, in Proposition 3.3 gives rise to an unramified surjective holomorphic map $\chi: T^{*}(B) \rightarrow M$ which commutes with the projection to $B$ and the map $f$. Then the pull-back of the symplectic form $\omega$ on $M$ by $\chi$ coincides with the standard symplectic structure of $T^{*}(B)$.

An immediate consequence is the following.

Proposition 3.6 Let $f: M \rightarrow B$ be a smooth Lagrangian fibration with a Lagrangian section $\Sigma \subset M$. Let $M^{\prime} \subset M$ be a family of abelian subvarieties of $f$, i.e., a submanifold containing $\Sigma$ such that $\left.f\right|_{M^{\prime}}$ is a smooth abelian fibration. Let $\mathcal{V} \subset T^{*}(B)$ be the subbundle defined by $\chi^{-1}\left(M^{\prime}\right)$. Then $\mathcal{V}$, regarded as a differential system on $B$, is involutive, i.e., the distribution $\mathcal{V}^{\perp} \subset T(B)$ annihilated by $\mathcal{V}$ is integrable.

Proof. By Proposition 3.5, the submanifold $\chi^{-1}(\Sigma) \subset T^{*}(B)$, i.e., the family of lattices for the family of abelian varieties, is Lagrangian in $T^{*}(B)$. The family of lattices for the family of abelian subvarieties $M^{\prime}$ is $\mathcal{V} \cap \chi^{-1}(\Sigma)$. Thus the subbundle $\mathcal{V}$ is locally generated by vectors lying in $\chi^{-1}(\Sigma)$. But Lagrangian sections of $T^{*}(B)$ are just closed 1 -forms on $B$. Thus the system $\mathcal{V}$ is locally generated by closed 1 -forms, and consequently it is involutive.

To use Proposition 3.6, we need to have a family of abelian subvarieties of a Lagrangian fibration. One construction leading to a family of abelian subvarieties is the following.

Proposition 3.7 Let $f: M \rightarrow B$ be a Lagrangian fibration with non-empty critical set $D \subset B$. Suppose that there exists a smooth proper morphism $\rho: B \rightarrow Z$ of relative dimension 1 onto a complex manifold $Z$. Assume that each fiber of $\rho$ intersects the critical set $D$. Then for each fiber $C$ of $\rho$, the abelian variety $A_{C}$ in the notation of Proposition 3.4 is of dimension $n-1$. In particular, if we choose an open subset $W \subset B \backslash D$ such that the restriction of $f$ to 
$f^{-1}(W), f^{-1}(W) \rightarrow W$, admits a Lagrangian section, then we have a family of $(n-1)$-dimensional abelian subvarieties in the smooth Lagrangian fibration $f^{-1}(W) \rightarrow W$, defined as the orbit of the Lagrangian section. The distribution on $W$ induced by this family of abelian subvarieties in the sense of Proposition 3.6 is just the fibers of $\rho$ on $W$.

Proof. For each $C, H^{0}\left(C, T^{*}(B)\right)$ contains the $(n-1)$-dimensional subspace corresponding to $\rho^{*} T_{\rho(C)}^{*}(Z)$. This subspace injects into $T_{e}\left(A_{C}\right)$ by the construction of the homomorphism $h_{C}$ in Proposition 3.4. Thus $A_{C}$ is an abelian variety of dimension $\geq n-1$. By Proposition 3.4 (iii), $A_{C}$ is an $(n-1)$-dimensional abelian variety. In particular, the homomorphism $h_{C}$ sends $T_{\rho(C)}^{*}(Z)$ onto $A_{C}$, which proves the last sentence of Proposition 3.7.

\section{Varieties of minimal rational tangents for the base man- ifold}

Now we go to the situation of Theorem 1.2. To start with, we recall the following result, which can be proved in various ways. The proof here was suggested by K. Oguiso.

Proposition 4.1 Let $f: M \rightarrow X$ be as in Theorem 1.2. Then the critical set $D$ is a non-empty hypersurface.

Proof. We already mentioned in Section 3 that $D$ is a hypersurface if it is non-empty. Suppose $D$ is empty. Then $f: M \rightarrow X$ is a proper smooth morphism. Denote by $\mathbf{Z}_{M}$ and $\mathbf{Z}_{X}$ the constant sheaves of the integer group on $M$ and $X$. Since $X$ is simply connected,

$$
R^{i} f_{*} \mathbf{Z}_{M} \cong \mathbf{Z}_{X}^{\oplus b_{i}(F)}
$$

where $b_{i}(F)$ denotes the $i$-th Betti number of a fiber $F$. From the beginning of the Leray spectral sequence for $f$, we get an exact sequence

$$
H^{1}\left(\mathbf{Z}_{M}\right) \longrightarrow H^{0}\left(R^{1} f_{*} \mathbf{Z}_{M}\right) \longrightarrow H^{2}\left(f_{*} \mathbf{Z}_{M}\right) .
$$

The first term vanishes because $M$ is simply connected. Consequently, $b_{1}(F) \leq b_{2}(X)$, which is absurd because $b_{1}(F)=2 n$ and $b_{2}(X)=1$.

Now let $X$ be as in Theorem 1.2. By Theorem 1.1, the base manifold $X$ is a Fano manifold with $b_{2}(X)=b_{4}(X)=1$ and $f$ is a Lagrangian fibration. Fix a minimal component $\mathcal{K}$ of $X$ and let $C$ be a general member of $\mathcal{K}$. We want to apply Proposition 3.4 to the complete curve $C \subset X$ and the Lagrangian fibration $f: M \rightarrow X$. However, we have little information about $H^{0}\left(C, T^{*}(X)\right)$. What we have is the information on $H^{0}\left(\hat{C}, \nu^{*} T^{*}(X)\right)$ where $\nu: \hat{C} \rightarrow C$ is the normalization. To remedy this, we lift the Lagrangian fibration $f$ to the normalization as follows.

Recall that $\nu: \hat{C} \rightarrow C \subset X$ is an immersion of $\mathbf{P}_{1}$. By analytic continuation, we can find an embedding of $\hat{C}$ in a complex manifold $B_{C}$ of dimension $n$ and a holomorphic map $\alpha: B_{C} \rightarrow M$ that is unramified at each point of $B_{C}$. The germ of $B_{C}$ along $\hat{C}$ is uniquely determined by the germ of $X$ along $C$. By pulling back the Lagrangian fibration $f: M \rightarrow X$ by the unramified holomorphic map $\alpha: B_{C} \rightarrow X$, we get a Lagrangian fibration

$$
f_{C}:=\alpha^{*} f: M_{C} \longrightarrow B_{C} .
$$


Now we apply Proposition 3.4 to $f_{C}$ and the complete curve $\hat{C} \subset B_{C}$. We have an abelian variety $A_{\hat{C}}$ acting on $f_{C}^{-1}(\hat{C})_{\text {red }}$ in the notation of Proposition 3.4.

Proposition 4.2 Let $f: M \rightarrow X$ be as in Theorem 1.2 and $\mathcal{K}$ be a minimal component on $X$. Let $\nu: \hat{C} \rightarrow C \subset X$ be the normalization of a general member of $\mathcal{K}$ and $\alpha: B_{C} \rightarrow X$ be the associated unramified holomorphic map as explained above. Let $f_{C}: M_{C} \rightarrow B_{C}$ be the pull-back of $f$ by $\nu$. Then

(1) $\operatorname{dim} A_{\hat{C}} \leq n-1$, and

(2) for a point $b \in \hat{C}$ outside the critical set and a point $z \in f_{C}^{-1}(b)$, the tangent space $T_{z}\left(A_{\hat{C}} \cdot z\right)$ contains the $\iota_{b, z}$-image of the evaluation of $H^{0}\left(\hat{C}, T^{*}\left(B_{C}\right)\right)=H^{0}\left(\hat{C}, \nu^{*} T^{*}(X)\right)$ at the point $b$.

Proof. Note that the critical set $D$ of $f$ is an ample hypersurface as a consequence of the condition $b_{2}(X)=1$ and Proposition 4.1. Thus $C$ intersects the critical set $D$ of $f$ and $\hat{C}$ intersects the critical set of $f_{C}$. Thus (1) and (2) follow from Proposition 3.4 (iii) and (iv).

Proposition 4.3 Let $f: M \rightarrow X$ be as in Theorem 1.2. For any minimal component $\mathcal{K}$ on $X$, the variety of minimal rational tangents $\mathcal{C}_{x}$ for a general point $x \in X$ is linear, i.e., each of its components is a linear subspace in $\mathbf{P} T_{x}(X)$.

Proof. Fix a general point $x \in X$ and a component $\mathcal{C}_{1}$ of the variety of minimal rational tangents $\mathcal{C}_{x} \subset \mathbf{P} T_{x}(X)$. Let $\mathcal{K}_{1}$ be the corresponding component of $\mathcal{K}_{x}$. For a general member $C$ of $\mathcal{K}_{1}, C$ is non-singular at $x$. Choose a point $z \in f_{C}^{-1}(x)$. For simplicity, we will identify $f_{C}^{-1}(x)$ and $f^{-1}(x)$ by the obvious isomorphism. By Proposition $4.2(2)$, the image in $T_{x}^{*}(X)$ of the evaluation of $H^{0}\left(\hat{C}, \nu^{*} T^{*}(X)\right)$ at $x$ is contained in $\iota_{x, z}^{-1}\left(T_{z}\left(A_{\hat{C}} \cdot z\right)\right)$ in the notation of Proposition 3.4 (iv). Now let us vary the general member $C$ of $\mathcal{K}_{1}$. The abelian subvariety $A_{\hat{C}} \cdot z$ of the abelian variety $f^{-1}(x)$ with $z$ as the origin remains unchanged, because there is no continuous family of abelian subvarieties in a fixed abelian variety. Thus the image of the evaluation of $H^{0}\left(\hat{C}, \nu^{*} T(X)\right)$ is contained in a fixed linear subspace $\iota_{x, z}^{-1}\left(T_{z}\left(A_{\hat{C}} \cdot z\right)\right)$. This linear subspace has dimension $<n$ by Proposition 4.2 (1). Thus by Proposition 2.1 , the dual variety of the component $\mathcal{C}_{1}$ of $\mathcal{C}_{x}$ in $\mathbf{P} T_{x}^{*}(X)$ is degenerate. Now apply Proposition 2.2 to complete the proof.

By Proposition 4.3, to prove Theorem 1.2, we may assume that $X$ satisfies (Assumption) in Section 2. In particular, we have the family of $\mathbf{P}_{p+1}$ 's described in Proposition 2.4 and apply the results in Section 2. The main consequence is the following.

Proposition 4.4 Let $H \subset X$ be an irreducible hypersurface such that a minimal component $\mathcal{K}$ is multivalent on $H$. Then $H$ is not contained in the critical set $D$ of the Lagrangian fibration $f: M \rightarrow X$.

To prove Proposition 4.4, we need two elementary lemmata. First, some notation. Suppose that we are given an abelian variety $A$ acting on another abelian variety $A^{\prime}$ by a morphism $\beta: A \times A^{\prime} \rightarrow A^{\prime}$. Fixing a point $z \in A^{\prime}$, the orbit map $\beta(\cdot, z): A \rightarrow A^{\prime}$ induces a homomorphism $\beta_{*}: H_{1}(A, \mathbf{Z}) \rightarrow H_{1}\left(A^{\prime}, \mathbf{Z}\right)$. In fact, this homomorphism does not depend on the choice of $z$, because the translation on $A^{\prime}$ acts trivially on $H_{1}\left(A^{\prime}, \mathbf{Z}\right)$. We have the following two lemmata.

Lemma 4.5 Let $A_{1}$ and $A_{2}$ be two abelian varieties acting on an abelian variety $A^{\prime}$, by two morphisms $\beta_{1}: A_{1} \times A^{\prime} \rightarrow A^{\prime}$ and $\beta_{2}: A_{2} \times A^{\prime} \rightarrow A^{\prime}$. Suppose that for a point $z \in A^{\prime}$, the tangents 
to the orbits $T_{z}\left(A_{1} \cdot z\right)$ and $T_{z}\left(A_{2} \cdot z\right)$ span $T_{z}\left(A^{\prime}\right)$. Then the images of the two homomorphisms

$$
\beta_{1 *}: H_{1}\left(A_{1}, \mathbf{Z}\right) \longrightarrow H_{1}\left(A^{\prime}, \mathbf{Z}\right) \text { and } \beta_{2 *}: H_{1}\left(A_{2}, \mathbf{Z}\right) \longrightarrow H_{1}\left(A^{\prime}, \mathbf{Z}\right)
$$

generate a subgroup of finite index in $H_{1}\left(A^{\prime}, \mathbf{Z}\right)$.

Proof. $\quad A_{1}^{\prime}:=A_{1} \cdot z$ and $A_{2}^{\prime}:=A_{2} \cdot z$ are abelian subvarieties in $A^{\prime}$. Since $T_{z}\left(A_{1}^{\prime}\right)$ and $T_{z}\left(A_{2}^{\prime}\right)$ span $T_{z}\left(A^{\prime}\right)$, there is a basis of $T_{z}\left(A^{\prime}\right)$ consisting of lattice vectors of $A_{1}^{\prime}$ and $A_{2}^{\prime}$ under the universal covering map $T_{z}\left(A^{\prime}\right) \rightarrow A^{\prime}$. Since the images of $\beta_{1 *}$ (resp. $\beta_{2 *}$ ) is of finite index in $H_{1}\left(A_{1}^{\prime}, \mathbf{Z}\right)$ (resp. $\left.H_{1}\left(A_{2}^{\prime}, \mathbf{Z}\right)\right)$, Lemma 4.5 follows.

Lemma 4.6 Let $f: \mathcal{A} \rightarrow B$ be a smooth abelian fibration. Assume that there exists an effective fiberwise action of an abelian variety $A$ on $\mathcal{A}$ given by $\beta: A \times \mathcal{A} \rightarrow \mathcal{A}$. For any $b \in B$, let $\beta(b): A \times f^{-1}(b) \rightarrow f^{-1}(b)$ be the action on the fiber and let

$$
\beta(b)_{*}: H_{1}(A, \mathbf{Z}) \longrightarrow H_{1}\left(f^{-1}(b), \mathbf{Z}\right)
$$

be the homomorphism induced by $\beta(b)$. Then the image of $\beta(b)_{*}$ is fixed under the monodromy action of $\pi_{1}(B, b)$ on $H_{1}\left(f^{-1}(b), \mathbf{Z}\right)$.

Proof. As $b$ varies over $B, \beta(b)_{*}$ defines a homomorphism from the constant system $H_{1}(A, \mathbf{Z})$ on $B$ into the local system defined by $H_{1}\left(f^{-1}(b), \mathbf{Z}\right)$ on $B$. Thus the image is a constant system, unchanged under the monodromy.

Proof of Proposition 4.4. Let us use the notation of Proposition 2.6. Let $y \in H$ be a general point of $H$ and choose neighborhoods $W \subset W_{0}$ as in Proposition 2.6. For a general point $x \in W \backslash H$, we get $\zeta_{1}, \zeta_{2} \in Z_{o}$ satisfying the properties in Proposition 2.6. For each $i=1,2$, choose a neighborhood $U_{i}$ of $\zeta_{i}$ in $Z$ such that $\mu$ is unramified on $B_{i}:=\rho^{-1}\left(U_{i}\right)$. Pulling back $f: M \rightarrow X$ by the unramified map $\left.\mu\right|_{B_{i}}$, we have a Lagrangian fibration $f_{i}: M_{i} \rightarrow B_{i}$. Applying Proposition 3.4 to $f_{i}$ and the complete variety $\rho^{-1}\left(\zeta_{i}\right)$, we get an action of an abelian variety $A_{i}$ on $f_{i}^{-1}\left(\rho^{-1}\left(\zeta_{i}\right)\right)_{\text {red. }}$. Note that the loop $\gamma_{i}$ on the smooth locus of $P_{\zeta_{i}}$ is naturally lifted to a loop in $\rho^{-1}\left(\zeta_{i}\right)$, which we denote by the same symbol $\gamma_{i}$. Pick a point $z \in f^{-1}(x)$. Let $x_{i} \in \rho^{-1}\left(\zeta_{i}\right)$ be the point over $x$ and $z_{i} \in f_{i}^{-1}\left(x_{i}\right)$ be a point on the fiber corresponding to $z$. The monodromy of the smooth Lagrangian fibration along $\gamma_{i}$ fixes the image of $H_{1}\left(A_{i}, \mathbf{Z}\right)$ in

$$
H_{1}\left(f_{i}^{-1}\left(x_{i}\right), \mathbf{Z}\right)=H_{1}\left(f^{-1}(x), \mathbf{Z}\right)
$$

by Lemma 4.6. But the two subspaces $\iota_{x, z}^{-1}\left(T_{z}\left(A_{1} \cdot z\right)\right)$ and $\iota_{x, z}^{-1}\left(T_{z}\left(A_{2} \cdot z\right)\right)$ span the whole $T_{x}^{*}(X)$ by Proposition 2.5 and Proposition $4.2(2)$. It follows that the monodromy action of $\pi_{1}\left(W_{0} \backslash H\right)$ on $H_{1}\left(f^{-1}(x), \mathbf{Z}\right)$ is trivial by Lemma 4.5. Thus the hypersurface is not in the critical set by Proposition 3.2.

An immediate consequence of Proposition 2.8, Proposition 4.1 and Proposition 4.4 is the following.

Proposition 4.7 Let $f: M \rightarrow X$ be as in Theorem 1.2. Suppose $X$ is different from $\mathbf{P}_{n}$. Then for any minimal component $\mathcal{K}, p=\operatorname{dim} \mathcal{C}_{x}=0$. 


\section{Use of the integrability of the distribution defined by a pair of rational curves}

By Proposition 4.7, to prove Theorem 1.2, we may assume that the base manifold $X$ has a minimal component $\mathcal{K}$ with $p=0$. We will make this assumption throughout this section. We start with the following observation.

Proposition 5.1 Let $X$ be as above with $p=0$. Let $\mu: X^{\prime} \rightarrow X$ be as in Proposition 2.4. If $E \subset X$ is a component of the branch locus of $\mu$, then $E$ is contained in the critical set $D$ of $f: M \rightarrow X$.

Proof. Suppose not. Let $y$ be a general point of $E$ outside the critical set $D$. Let $R$ be a component of the ramification locus of $\mu$ such that $\mu(R)=E$. Let $z \in R$ be a point in $\mu^{-1}(y)$. We can choose an open neighborhood $W^{\prime}$ of $z$ and an open neighborhood $W$ of $y$ with $W \cap D=\emptyset$ such that

(i) $W$ and $W^{\prime}$ are biholomorphic to a polydisc with $E \cap W \subset W$ and $R \cap W^{\prime} \subset W^{\prime}$ biholomorphic to the coordinate hyperplane;

(ii) there exists a Lagrangian section $\Sigma \subset f^{-1}(W)$ of $f$ over $W$;

(iii) $W^{\prime} \backslash R \subset \rho^{-1}\left(Z_{o}\right)$; and

(iv) $\left.\mu\right|_{W^{\prime}}: W^{\prime} \rightarrow W$ is a cyclic branched covering of degree $>1$.

Pulling back $f$ by the unramified holomorphic map $\left.\mu\right|_{W^{\prime} \backslash R}$, we get a smooth Lagrangian fibration $f^{\prime}: M^{\prime} \rightarrow W^{\prime} \backslash R$ with a Lagrangian section $\Sigma^{\prime} \subset M^{\prime}$. Applying Proposition 3.7, we get a family of abelian subvarieties of dimension $n-1$, say $\mathcal{A} \subset M^{\prime}$, over $W^{\prime} \backslash R$. By Proposition 2.4 (c), we know that the fibers of $\rho$ on $W^{\prime} \backslash R$ are sent to $W \backslash E$ to define a multi-valued foliation. The relation between the family of abelian subvarieties $\mathcal{A}$ and the fibers of $\rho$ in Proposition 3.7 implies that the image of $\mathcal{A}$ in $f^{-1}(W \backslash E)$ is a multi-valued family of abelian subvarieties. Thus the Galois group of the cyclic covering $\left.\mu\right|_{W^{\prime}}$ acts non-trivially on the family of lattices for $\mathcal{A}$. This means that the monodromy of $\pi_{1}(W \backslash E)$ acts non-trivially on $H_{1}\left(f^{-1}(b)\right.$, Z $)$ for a point $b \in W \backslash E$. This is absurd because the Lagrangian fibration $f$ is smooth over $W$.

Now we introduce a new notion. The family $Z$ in Proposition 2.4 is a $\mathbf{P}_{1}$-bundle over an open subset $Z_{o}$ of $Z$. Let $x \in X$ be a general point so that all members of $\mathcal{K}_{x}$ are smooth at $x$ and have distinct tangent vectors at $x$ by Proposition 2.5. Furthermore, we can assume that $x$ lies outside $\mu\left(\rho^{-1}\left(Z \backslash Z_{o}\right)\right)$ and the branch locus of $\mu$. Let $\zeta_{1}, \zeta_{2} \in Z_{o}$ be two distinct points with $x \in P_{\zeta_{1}} \cap P_{\zeta_{2}}$. Let $y_{1}=\rho^{-1}\left(\zeta_{1}\right) \cap \mu^{-1}(x)$ and $y_{2}=\rho^{-1}\left(\zeta_{2}\right) \cap \mu^{-1}(x)$. Choose a small neighborhood $W$ of $x, W_{1}$ of $y_{1}$ and $W_{2}$ of $y_{2}$ such that

(i) $W_{1}, W_{2}$ and $W$ are biholomorphic to one another by $\mu$;

(ii) fibers of $\rho$ in $W_{1}$ and fibers of $\rho$ in $W_{2}$ induce two transversal holomorphic foliations of rank 1 on $W$.

The span of these two foliations define a distribution of rank 2 on $W$, which we denote by $\mathcal{D}$. We call $\mathcal{D}$ the distribution defined by the pair $P_{\zeta_{1}}$ and $P_{\zeta_{2}}$.

Proposition 5.2 Let $X$ be a Fano manifold with $b_{2}(X)=1$ having a minimal component $\mathcal{K}$ with $p=0$. Let $x \in X$ be a general point and $C_{1}, C_{2}$ be two distinct members of $\mathcal{K}_{x}$. If $X$ is a base manifold of a fibration $f: M \rightarrow X$ as in Theorem 1.2, then the distribution $\mathcal{D}$ defined by the pair $C_{1}$ and $C_{2}$ in a neighborhood of $x$ is integrable. 
Proof. Let $B:=\rho^{-1}\left(Z_{o}\right)$, then $\mu$ is unramified on $B$. Pulling back $f: M \rightarrow X$ by $\mu$, we get a Lagrangian fibration $g: M^{\prime} \rightarrow B$ and a smooth fibration by curves $\rho: B \rightarrow Z_{o}$. Let $W$ be a neighborhood of $x$ and $W_{1}, W_{2} \subset B$ be the open subsets used in the definition of $\mathcal{D}$. By shrinking $W$ if necessary, we may assume that $f$ has a Lagrangian section over $W$, hence the induced Lagrangian fibrations over $W_{1}$ and $W_{2}$ also have Lagrangian sections. Applying Proposition 3.7 to $W_{1}$ and $W_{2}$, we get two families $\mathcal{A}_{1}$ and $\mathcal{A}_{2}$ of abelian subvarieties of dimension $n-1$ inside the Lagrangian fibration $\left.f\right|_{f^{-1}(W)}$. The annihilator $\mathcal{D}^{\perp} \subset T^{*}(W)$ of the distribution $\mathcal{D} \subset T(W)$ corresponds, in the sense of Proposition 3.6, to the family of abelian subvarieties of dimension $n-2$ defined by the intersection $\mathcal{A}_{1} \cap \mathcal{A}_{2}$. Thus $\mathcal{D}$ is integrable by Proposition 3.6.

Proposition 5.3 Let us assume the situation of Proposition 5.2 and use the notation of Proposition 2.4. Given a general member $C$ of $\mathcal{K}$, let $C^{\prime}$ be a component of $\mu^{-1}(C)$ such that

$$
\left.\mu\right|_{C^{\prime}}: C^{\prime} \longrightarrow C
$$

is finite of degree $>1$, which exists by Proposition 2.3. Let

$$
h: \hat{C}^{\prime} \longrightarrow \rho \widehat{\left(C^{\prime}\right)}
$$

be the lift of

$$
\left.\rho\right|_{C^{\prime}}: C^{\prime} \longrightarrow \rho\left(C^{\prime}\right)
$$

to the normalizations of $C^{\prime}$ and $\rho\left(C^{\prime}\right)$. Then $h$ has a ramification point $z \in \hat{C}^{\prime}$ such that the image of $h(z)$ in $\rho\left(C^{\prime}\right)$ lies in $Z_{o}$.

We need two lemmata.

Lemma 5.4 Let $E$ be a component of the branch divisor of $\mu$. Let $E^{\prime}$ be an irreducible component of $\mu^{-1}(E)$ which is dominant over $\mathcal{K}$ by $\rho$. Then $C^{\prime}$ is disjoint from $E^{\prime}$.

Proof. Otherwise, through a general point $x^{\prime}$ of $E^{\prime}$, we have two distinct curves, $C^{\prime}$ and a fiber of $\rho$, neither of which are contained in $E^{\prime}$. Since $\mu$ is unramified at $x^{\prime}$ by Proposition 2.4, the images of these curves under $\mu$ are of the form $P_{\zeta_{1}}, P_{\zeta_{2}}$ with $\zeta_{1} \neq \zeta_{2}$. Since these two curves pass through $x=\mu\left(x^{\prime}\right)$, which is a general point of $E, \mathcal{K}$ is multivalent on $E$. Then $E$ is not in the critical set of $f$ by Proposition 4.4, a contradiction to Proposition 5.1.

Lemma 5.5 There exist a family of members of $\mathcal{K}$

$$
\left\{C_{t}, t \in \Delta, C=C_{0}\right\}
$$

and the associated deformation

$$
\left\{C_{t}^{\prime}, t \in \Delta, C^{\prime}=C_{0}^{\prime}\right\}
$$

such that for each $t \in \Delta$,

(i) $C_{t}^{\prime}$ is a component of $\mu^{-1}\left(C_{t}\right)$;

(ii) $\left.\mu\right|_{C_{t}^{\prime}}: C_{t}^{\prime} \rightarrow C_{t}$ is finite of degree $>1$ over $C_{t}$;

(iii) $\rho\left(C_{t}^{\prime}\right)=\rho\left(C^{\prime}\right)$.

Proof. Let $x \in C$ be a general point. Pick a point $x^{\prime} \in C^{\prime}$ with $\mu\left(x^{\prime}\right)=x$ and set $C^{x}:=$ $\mu\left(\rho^{-1}\left(\rho\left(x^{\prime}\right)\right)\right)$. Then $C^{x}$ is a member of $\mathcal{K}_{x}$. The triviality of the normal bundle of $C$ implies 
that there exists a 'deformation of $C$ along $C^{x}$, namely, a unique (up to reparametrization) deformation $\left\{C_{t}, t \in \Delta\right\}$ of $C=C_{0}$ satisfying $C_{t} \cap C^{x} \neq \emptyset$. More precisely, there exists a unique component $C^{\sharp}$ of $\mu^{-1}\left(C^{x}\right)$ different from $\rho^{-1}\left(\rho\left(x^{\prime}\right)\right)$ that contains the point $\rho^{-1}(\zeta) \cap \mu^{-1}(x)$ where $P_{\zeta}=C$. Then the germ of $\rho\left(C^{\sharp}\right)$ near $\zeta$ gives a deformation $\left\{C_{t}, t \in \Delta, C=C_{0}\right\}$ such that each $C_{t}$ belongs to $\mathcal{K}$ and $C_{t} \cap C^{x} \neq \emptyset$. The germ of such a deformation of $C$ is uniquely determined by $C^{x}$, up to reparametrization.

By the generality of the choice of $C$, we have the associated deformation $\left\{C_{t}^{\prime}, t \in \Delta\right\}$ of $C^{\prime}=C_{0}^{\prime}$ such that $\left.\mu\right|_{C_{t}^{\prime}}: C_{t}^{\prime} \rightarrow C_{t}$ is finite of degree $>1$ for any $t \in \Delta$.

It remains to show that $\rho\left(C_{t}^{\prime}\right)=\rho\left(C^{\prime}\right)$ for each $t \in \Delta$. This follows from the fact that the leaf through $x$ of the distribution $\mathcal{D}$ defined by $C$ and $C^{x}$ gives the germ of the surface traced out by the deformation of $C$ along $C^{x}$ and at the same time the germ of the surface traced out by the deformation of $C^{x}$ along $C$. More precisely, it suffices to show that

$$
\text { (*) } \rho^{-1}(z) \cap C_{t}^{\prime} \neq \emptyset \text { for each } z \in \rho\left(C^{\prime}\right) \text { and each } t \in \Delta \text {. }
$$

Clearly $\rho^{-1}\left(\rho\left(x^{\prime}\right)\right) \cap C_{t}^{\prime} \neq \emptyset$. Suppose that we vary the choice of $x \in C$ in the above construction of $C_{t}$, say $\left\{x_{s} \in C, s \in \Delta, x_{0}=x\right\}$. Let $x_{s}^{\prime} \in C^{\prime}$ be the associated variation of $x^{\prime}=x_{0}$ and $C^{x_{s}}:=$ $\mu\left(\rho^{-1}\left(\rho\left(x_{s}\right)\right)\right.$. By Proposition 5.2, the deformation of $C$ along $C^{x_{s}}$ are, up to reparametrization, the same as the deformation of $C$ along $C^{x}$ for any $s \in \Delta$. Thus $\rho^{-1}\left(\rho\left(x_{s}^{\prime}\right)\right) \cap C_{t}^{\prime} \neq \emptyset$ for each $s \in \Delta$. This proves $(*)$ for $z$ in a neighborhood of $\rho\left(x^{\prime}\right)$. It follows that $(*)$ holds for all $z \in \rho\left(C^{\prime}\right)$.

Proof of Proposition 5.3. Suppose that $h$ is unramified over $\rho\left(C^{\prime}\right) \cap Z_{o}$. Let us use the deformation $C_{t}$ constructed in Lemma 5.5. By the generality of $C$, we may assume that for each $t \in \Delta$ the holomorphic map

$$
h_{t}: \hat{C}_{t}^{\prime} \rightarrow \rho \widehat{\left(C_{t}^{\prime}\right)}=\rho \widehat{\left(C^{\prime}\right)}, \quad h_{0}=h
$$

which is the lift of $\left.\rho\right|_{C_{t}^{\prime}}$ to the normalization of curves, is unramified over $\rho\left(C^{\prime}\right) \cap Z_{o}$. Since $h_{t}$ is a continuous family of coverings of the Riemann surface $\rho \widehat{\left(C^{\prime}\right)}$ with fixed branch locus, we can find a biholomorphic map

$$
\text { (\$) } \psi_{t}: \hat{C}^{\prime} \rightarrow \hat{C}_{t}^{\prime}, \quad \psi_{0}=\operatorname{Id}_{\hat{C}^{\prime}} \text { with } h=h_{t} \circ \psi_{t} \text {, }
$$

which depends holomorphically on $t$ (e.g. [Sh, p. 32, Corollary 1].).

From Lemma 5.5 (ii), there are at least two distinct points in $\hat{C}_{t}$, say $a_{t} \neq b_{t} \in \hat{C}_{t}$, such that the corresponding points in $C_{t}$ lie in the branch divisor of $\mu$ in $X$. Let $\{0, \infty\} \subset \mathbf{P}_{1}$ be two distinct points on the projective line. We can choose a family of biholomorphic maps $\left\{\sigma_{t}: \hat{C}_{t} \rightarrow \mathbf{P}_{1}, t \in \Delta\right\}$ such that $\sigma_{t}\left(a_{t}\right)=0$ and $\sigma_{t}\left(b_{t}\right)=\infty$ for each $t \in \Delta$. Denote by $\mu_{t}: \hat{C}_{t}^{\prime} \rightarrow \hat{C}_{t}$ the lift of $\left.\mu\right|_{C_{t}^{\prime}}$ to the normalization of curves. Then

$$
\left\{\varphi_{t}: \hat{C}^{\prime} \longrightarrow \mathbf{P}_{1}, \quad \varphi_{t}:=\sigma_{t} \circ \mu_{t} \circ \psi_{t}, t \in \Delta\right\}
$$

is a family of meromorphic functions on the compact Riemann surface $\hat{C}^{\prime}$. 
By Lemma 5.4, for each component $E$ of the branch divisor of $\mu$, the intersection of $C_{t}^{\prime}$ with $\mu^{-1}(E)$ has a fixed image in $\rho\left(C^{\prime}\right)=\rho\left(C_{t}^{\prime}\right)$, independent of $t \in \Delta$. This implies that there is a finite subset $Q \subset \rho\left(\widehat{C^{\prime}}\right)$, independent of $t$, such that

$$
\mu_{t}^{-1}\left(a_{t}\right) \cup \mu_{t}^{-1}\left(b_{t}\right) \subset h_{t}^{-1}(Q)
$$

for any $t \in \Delta$. Then

$$
\varphi_{t}^{-1}(0)=\psi_{t}^{-1} \circ \mu_{t}^{-1} \circ \sigma_{t}^{-1}(0)=\psi_{t}^{-1}\left(\mu_{t}^{-1}\left(a_{t}\right)\right) \subset \psi_{t}^{-1}\left(h_{t}^{-1}(Q)\right)
$$

for all $t \in \Delta$. Since $\psi_{t}^{-1}\left(h_{t}^{-1}(Q)\right)=h^{-1}(Q)$ by the choice of $\psi_{t}$ in $(\boldsymbol{\beta}), \varphi_{t}^{-1}(0) \subset h^{-1}(Q)$ for any $t \in \Delta$. Consequently, $\varphi_{t}^{-1}(0)=\varphi_{0}^{-1}(0)$ for all $t \in \Delta$. By the same argument we get $\varphi_{t}^{-1}(\infty)=\varphi_{0}^{-1}(\infty)$ for all $t \in \Delta$. In other words, the family of meromorphic functions $\varphi_{t}$ have the same zeroes and the same poles on the Riemann surface $\hat{C}^{\prime}$. This implies that for any $z \in \mathbf{P}_{1}$ and $t \in \Delta, \varphi_{t}^{-1}(z)=\varphi_{0}^{-1}(z)$. It follows that for any $w_{1}, w_{2} \in \hat{C}^{\prime}$ and any $t \in \Delta$,

$$
(\diamond) \quad \varphi_{t}\left(w_{1}\right)=\varphi_{t}\left(w_{2}\right) \text { if and only if } \varphi_{0}\left(w_{1}\right)=\varphi_{0}\left(w_{2}\right) .
$$

Since $\left.\mu\right|_{C^{\prime}}$ is finite of degree $>1$ by our assumption, we can choose two points $\alpha \neq \beta \in \hat{C}^{\prime}$ such that $\varphi_{0}(\alpha)=\varphi_{0}(\beta)$. Furthermore, denoting by $\bar{\alpha} \in \rho\left(C^{\prime}\right)$ (resp. $\bar{\beta} \in \rho\left(C^{\prime}\right)$ ) the point corresponding to $h_{0}(\alpha) \in \rho \widehat{\left(C^{\prime}\right)}$ (resp. $\left.h_{0}(\beta) \in \rho\left(\widehat{C^{\prime}}\right)\right)$ under the normalization, we may assume that

$(\nabla) \bar{\alpha}$ and $\bar{\beta}$ are two distinct points in $Z_{o}$.

From $(\diamond)$, we have $\varphi_{t}(\alpha)=\varphi_{t}(\beta)$ for all $t \in \Delta$. Since $\varphi_{t}=\sigma_{t} \circ \mu_{t} \circ \psi_{t}$ and $\sigma_{t}$ is biholomorphic, we see that

(ه) $\mu_{t} \circ \psi_{t}(\alpha)=\mu_{t} \circ \psi_{t}(\beta)$ for all $t \in \Delta$.

Denote by

$$
\alpha_{t} \in C_{t}^{\prime} \subset X^{\prime} \quad\left(\text { resp. } \beta_{t} \in C_{t}^{\prime} \subset X^{\prime}\right)
$$

the point corresponding to $\psi_{t}(\alpha) \in \hat{C}_{t}^{\prime}\left(\right.$ resp. $\left.\psi_{t}(\beta) \in \hat{C}_{t}^{\prime}\right)$ under the normalization. Then the locus

$$
A:=\left\{\alpha_{t} \in X^{\prime}, t \in \Delta\right\} \quad\left(\text { resp. } B:=\left\{\beta_{t} \in X^{\prime}, t \in \Delta\right\}\right)
$$

covers a non-empty open subset in the fibre $\rho^{-1}(\bar{\alpha})$ (resp. $\rho^{-1}(\bar{\beta})$ ). Thus $\mu(A)$ (resp. $\mu(B)$ ) covers a non-empty open subset in

$$
P_{\bar{\alpha}}:=\mu\left(\rho^{-1}(\bar{\alpha})\right) \quad\left(\text { resp. } P_{\bar{\beta}}:=\mu\left(\rho^{-1}(\bar{\beta})\right)\right) .
$$

Since $\mu(A)$ (resp. $\mu(B)$ ) is the locus of points corresponding to $\mu_{t} \circ \psi_{t}(\alpha)$ (resp. $\mu_{t} \circ \psi_{t}(\beta)$ ) by the normalization $\hat{C}_{t} \rightarrow C_{t}$, the equality $(\boldsymbol{N})$ above implies that $\mu(A)=\mu(B)$. Consequently,

$$
P_{\bar{\alpha}}=P_{\bar{\beta}},
$$

a contradiction to $(\mathcal{Q})$ and Proposition 2.4 (c).

Proposition 5.6 In the situation of Proposition 5.3, there exists an irreducible hypersurface $H \subset X$ such that through a general point $x \in H$, there are two distinct members $C_{1}$ and $C_{2}$ of 
$\mathcal{K}$ with $T_{x}\left(C_{1}\right)=T_{x}\left(C_{2}\right)$ in $T_{x}(X)$ where $T_{x}\left(C_{1}\right)$ (resp. $T_{x}\left(C_{2}\right)$ ) denotes the tangent space at $x$ of a component of the germ of $C_{1}$ (resp. $C_{2}$ ) at $x$. In particular, $\mathcal{K}$ is multivalent on $H$.

Proof. In the situation of Proposition 5.3, let $z \in C^{\prime}$ be the image of a ramification point of $h$ such that $\rho(z) \in Z_{o}$. Then $\mu$ is unramified at $z$ and the curve $\mu\left(\rho^{-1}(\rho(z))\right)$ in $X$ is an immersed $\mathbf{P}_{1}$. It follows that $C^{\prime}$ is immersed at $z$ and one of the component of the germ of $C^{\prime}$ at $z$ must be tangent to $\rho^{-1}(\rho(z))$ because $z$ is a ramification point of $h$. But then the two members $C$ and $\mu\left(\rho^{-1}(\rho(z))\right)$ of $\mathcal{K}$ have non-empty intersection at $\mu(z)$, sharing a common tangent. As $C$ varies, this intersection point also varies to define a hypersurface $H$. This proves Proposition 5.6.

Finally, the next proposition leads to a contradiction with Proposition 4.4 and Proposition 5.6, completing the proof of Theorem 1.2. $f$.

Proposition 5.7 The hypersurface $H$ in Proposition 5.6 is contained in the critical set $D$ of

Proof. Suppose not. Let $y \in H$ be a general point. By the definition of $H$, there are two points $y_{1}, y_{2} \in \mu^{-1}(y)$ where $\mu$ is unramified, such that $P_{\zeta_{1}}, \zeta_{1}=\rho\left(y_{1}\right)$, and $P_{\zeta_{2}}, \zeta_{2}=\rho\left(y_{2}\right)$ are two distinct members of $\mathcal{K}$ tangent to each other at $y$. Choose a neighborhood $W$ of $y$ and a neighborhood $W_{i}$ of $y_{i}$ for each $i=1,2$ such that $W, W_{1}$ and $W_{2}$ are all biholomorphic by $\mu$. By shrinking $W$ if necessary, we can assume that $\left.f\right|_{f^{-1}(W)}$ is a smooth Lagrangian fibration with a Lagrangian section over $W$. The same holds for the pull-backs of $f$ to $W_{1}$ and $W_{2}$. Thus by Proposition 3.7, we get two families of abelian subvarieties of dimension $n-1$ in the Lagrangian fibration $f^{-1}(W) \rightarrow W$ such that each abelian subvariety in the fiber is tangent to the image of the conormal bundle of deformations of $C$ and $C^{\prime}$. Since $C$ and $C^{\prime}$ are tangent along $H$, the two families of abelian subvarieties coincide along $H$, but not at a general point of $W$. This is a contradiction to the following elementary lemma.

Lemma 5.8 Given a family of abelian variety $\mathcal{A} \rightarrow S$ and two families of abelian subvarieties $\mathcal{A}_{1} \rightarrow S$ and $\mathcal{A}_{2} \rightarrow S$, if the fibers of $\mathcal{A}_{1}$ and $\mathcal{A}_{2}$ coincide at some point of $S$, then $\mathcal{A}_{1}=\mathcal{A}_{2}$.

Proof. Consider the family of quotient abelian varieties $\mathcal{A} / \mathcal{A}_{1} \rightarrow S$ and the relative group quotient projection $\mathcal{A} \rightarrow \mathcal{A} / \mathcal{A}_{1}$. This projection sends one fiber of $\mathcal{A}_{2}$ to a point. So it must send each fiber of $\mathcal{A}_{2}$ to a point by [Mu, Proposition 6.1]. Thus $\mathcal{A}_{1}=\mathcal{A}_{2}$.

Acknowledgment It is a pleasure to thank Yasunari Nagai, Yong-Geun Oh, and Justin Sawon for beneficial discussions. I am particularly grateful to Daisuke Matsushita, who kindly sent me his preprint [Ma4] and explained several points of his works to me. A very special thank goes to Keiji Oguiso, for many discussions on holomorphic symplectic geometry and very valuable comments on the first draft of this paper.

\section{References}

[CMS] Cho, K., Miyaoka, Y. and Shepherd-Barron, N.: Characterizations of projective space and applications to complex symplectic manifolds. In Higher dimensional birational geometry (Kyoto, 1997), Adv. Stud. Pure Math. 35 (2002) 1-88

[GuSt] Guillemin, V. and Sternberg, S.: Symplectic techniques in physics. Second edition. Cambridge University Press, Cambridge, 1990 
[Hu] Huybrechts, D.: Compact hyperkähler manifolds. In Calabi-Yau manifolds and related geometries (Nordfjordeid, 2001), 161-225, Universitext, Springer, Berlin, 2003

[Hw] Hwang, J.-M.: Deformation of holomorphic maps onto Fano manifolds of second and fourth Betti numbers 1. Ann. Inst. Fourier 57 (2007) 815-823

[HwMo1] Hwang, J.-M. and Mok, N.: Cartan-Fubini type extension of holomorphic maps for Fano manifolds of Picard number 1. Journal Math. Pures Appl. 80 (2001) 563-575

[HwMo2] Hwang, J.-M. and Mok, N.: Birationality of the tangent map for minimal rational curves. Asian J. Math. 8, Special issue dedicated to Yum-Tong Siu, (2004) 51-64

[HwOg] Hwang, J.-M. and Oguiso, K.: Characteristic foliation on the discriminantal hypersurface of a holomorphic Lagrangian fibration. preprint (arXiv:0710.2376)

[HwRa] Hwang, J.-M. and Ramanan, S.: Hecke curves and Hitchin discriminant. Ann. scient. Ec. Norm. Sup. 37 (2004) 801-817

[Ka] Kaup, W.: Infinitesimale Transformationsgruppen komplexer Räume. Math. Annalen 160 (1965) 72-92

[Ma1] Matsushita, D.: On fibre space structures of a projective irreducible symplectic manifold. Topology 38 (1999), 79-83, and addendum: Topology 40 (2001) 431-432

[Ma2] Matsushita, D.: Equidimensionality of Lagrangian fibrations on holomorphic symplectic manifolds. Math. Res. Lett. 7 (2000) 389-391

[Ma3] Matsushita, D.: Higher direct images of dualizing sheaves of Lagrangian fibrations. Amer. J. Math. 127 (2005) 243-259

[Ma4] Matsushita, D.: A canonical bundle formula of projective Lagrangian fibrations. preprint (arXiv:0710.0122)

[Mu] Mumford, D. and Fogarty, J.: Geometric invariant theory. Second enlarged edition. Springer Verlag, 1982

[Ng] Nagai, Y.: Dual fibration of a projective Lagrangian fibration. preprint

[Nk] Nakayama, N.: Compact Kähler manifolds whose universal covering spaces are biholomorphic to $\mathbf{C}^{n}$. RIMS preprint 1230.

[Sh] Shokurov, V. V.: Riemann surfaces and algebraic curves. In Algebraic curves, algebraic manifolds and schemes, Springer Verlag, 1998

Jun-Muk Hwang

Korea Institute for Advanced Study

207-43 Cheongnyangni-dong

Seoul 130-722, Korea

jmhwang@kias.re.kr 